\title{
Integrated Pitch-Yaw Acceleration Autopilot Design for Varying-Velocity Man Portable Missile
}

\author{
A. Awad and H. P. Wang
}

\begin{abstract}
A design of integrated pitch-yaw acceleration autopilot based on sliding mode control is proposed for roll-stabilized skid-to-turn (STT) varying-velocity man portable missile. This approach is suggested to achieve a good tracking performance in the presence of nonlinear aerodynamics, command dynamics, and missile parameters' variation during the entire flight envelope, including boosting phase, sustaining phase, and gliding phase where fast variation of velocity and parameters exists. Thus, robustness against quick variation of velocity and parameters is exceedingly required. The presented autopilot consists of two-loop structure with an additional proportional gain that switches according to time of the boosting phase, controls STT maneuver. It employs multiple sliding surfaces to generate control signals for pitch-yaw channels simultaneously. The missile model considers the velocity variation, the gravity effect, the missile parameters' variation, and the environmental conditions. Finally, Numerical simulation is established to evaluate performance of the proposed acceleration autopilot.
\end{abstract}

Index Terms-Acceleration autopilot, flight control system, man portable missile, sliding mode control.

\section{INTRODUCTION}

Missile autopilot design is still considered as one of the most attractive topics for control engineers due to its enormous nonlinear dynamics, and its rapid parameters' variation [1]. The most significant variation of missile parameters is the missile velocity which changes rapidly as a result of missile subjection to sudden acceleration during boosting phase and deceleration in gliding phase due to aerodynamic drag [2]. One of the important air defense systems is the man-portable missile system in which the missile should be launched in low-speed and that results in a dramatic parameters' variation. Nevertheless, the fin deflection angles are limited because of the hardware constraints [3]. It is clear from the above that, the missile autopilot design faces a big challenges, especially in the man portable missile type [1], [3].

Manuscript received November 27, 2015; revised February 20, 2016. This work was partially supported by the National Natural Science Foundation of China $(61304077,61203115)$, by the Natural Science Foundation of Jiangsu Province (BK20130765), by the Specialized Research Fund for the Doctoral Program of Higher Education of China (20123219120038), by the Chinese Ministry of Education Project of Humanities and Social Sciences (13YJCZH171), by the Fundamental Research Funds for the Central Universities (30920130111014), and by the Zijin Intelligent Program of Nanjing University of Science and Technology (2013_ZJ_0105).

A. Awad and H. P. Wang are with Sino-French International Joint Laboratory of Automatic Control and Signal Processing (LaFCAS), Automation School, Nanjing University of Science and Technology, China (e-mail: ahmed_eyssa@hotmail.com,hp.wang@njust.edu.cn).
The work in this field had been started since 1944's [4]. One of the commonly used autopilots was the three-loop autopilot topology [4]. The conventional and linear quadratic regulators based on the linearization of model dynamics around fixed operating points have been used. The autopilot produced from the fixed-point regulators' interpolation, is so-called "gain scheduling,". In [5] a classical gain-scheduling design, was introduced. In the 1990's, extensions of these techniques had brought many developments, like guaranteed stability margins and performance levels [6], [7]. Also, robustness issues had been introduced with suitable extensions of $H_{\infty}$ techniques [8], [9]. The gain scheduling technique shows a good performance but it guarantees global stability only in case of slow variation of both the states and the missile parameters.

The development of linear parameter-varying (LPV) and quasi-LPV approaches in the last two decades had pushed the researchers towards a new, systematic, and strict methodology. Unfortunately, the disadvantage of these approaches, especially for quasi-LPV autopilot, is the increment of conservative level [10]. An acceleration autopilot design using the LPV reference model was presented for portable missile in [3]. Most of these approaches except quasi-LPV approach, were still based on the linearization of dynamics around operating points. Besides, other drawbacks of LPV were the difficulty of parameter variation recognition, and the demand of an additional filter for parameter estimation as well [11].

The requirements of high maneuverability and the development of nonlinear control methods, pushed the research towards new control design approaches that consider essential nonlinear dynamics. This led to the first generation of nonlinear autopilots which were based on both the inversion of dynamics [12], and the feedback linearization techniques [13]. New approaches were introduced in the last decades based on recent control techniques: such as Lyapunov stabilization techniques [14], $l_{1}$ adaptive control [15], simple adaptive control algorithm [16], immersion and invariance control [17], backstepping control [18], and state-dependent Riccati equation approach [19]. Also, one of the control techniques commonly used in missile autopilot design, was sliding mode control (SMC) [20]. The approaches introduced in nonlinear and/or adaptive context failed when massive dynamics existed. Moreover, the strict requirements on the response speed cannot often be achieved due to adaptation laws. Also, it should be mentioned that though the approaches based on these methods demonstrate a good performance but it have been only applied on simple single-input/single-output cases, disregarding variation of the missile velocity, and its 
parameters and the nonlinearities occurring between pitch and yaw planes.

Finally, there are a few researches deal with the autopilot design in multi-input/multi-output (MIMO) way. For example, A robust backstepping approach has been applied for MIMO model to achieve both bank-to-turn and STT maneuvers [1], and acceleration autopilot based on a linear robust control scheme was presented to control roll, pitch, and yaw channels in an integrated way [21]. These works show a good performance whereas considering the missile velocity constant. In [2] a sliding mode based roll-pitch-yaw integrated attitude and acceleration autopilot for a time-varying velocity STT missile was proposed. It showed a good performance but it considered the velocity variation as a function of time. Furthermore, it neglected the gravity effect, chattering phenomenon, and missile parameters' variation.

This paper has developed the SMC based acceleration autopilot, which was presented in the literature considering the above referred neglected factors, and more missile dynamic parameters in MIMO way. Namely, most of the previous works omitted the gravity effect, and considered the missile parameters (velocity, thrust, mass, moments of inertia, and environmental conditions) constant. In this paper, the missile model has accounted the gravity effect, the missile parameters' dynamics, and the nonlinear aerodynamics. In a similar manner, the missile velocity and height have been considered as a function of its states. Also, the dynamic acceleration commands in boosting phase are regarded in the simulation results. multiple sliding surfaces are proposed to deal with the model characteristics' complexity and a new switched proportional gain is added to the autopilot structure to improve its tracking performance and to get over the inconvenient effects resulting from the aforementioned considerations in missile model. This new switched gain is changed according to time of the boosting phase. Finally, The closed loop stability is discussed as well.

This paper is organized as follows. In Section II, system modeling is provided. In Section III, pith-yaw acceleration autopilot design, and its stability studying are presented. In Section IV, numerical simulations are presented, and Section $\mathrm{V}$ is devoted to summary and concluding remarks.

\section{SySTEM DESCRIPTION AND MODELING}

The missile of concern is a roll-stabilized STT varying-velocity man portable missile, and it is aerodynamically controlled via canard fins. Thus, the next general assumptions can be considered:

1) Missile has an axis-symmetric, and cruciform shape so that the moments of inertia $I y y(t)$ and $I z z(t)$ are identical and products of inertia moments can be discarded.

2) For short-range missiles, The earth has been considered flat, and non-rotating.

3) Roll autopilot is designed separately to achieve: the missile roll turning rate $p=0$, and the roll angle $\varphi=0$.

4) Missile speed Vm can be measured.

\section{A. Mathematical Modeling}

As a result of missile states, and its dynamic parameters measured in different coordinates; orientation of the common used coordinate systems, and the related angles between them are depicted in Fig. 1. The concerned missile motion in space is described by means of the following differential equations. Solution of these equations gives missile linear velocity components $(u, v, w)$ in $\left(X_{b}, Y_{b}, Z_{b}\right)$ axes of body coordinate system (BCS), respectively, missile angular rates $(q, r)$ around $\left(Y_{b}, Z_{b}\right)$ axes, respectively, the pitch Euler angle $\theta$, the angle of attack, and the side slip angle $(\alpha, \beta)$, and the missile height $h$ in $Z_{i}$ axis of inertial coordinate system (ICS). The differential equations, $V_{m}$, dynamic pressure $Q\left(h, V_{m}\right)$, and force components $\left(F_{x b}, F_{y b}, F_{z b}\right)$ applied on the missile in $\left(X_{b}\right.$, $\left.Y_{b}, Z_{b}\right)$, respectively are expressed as follows [22]-[24]:

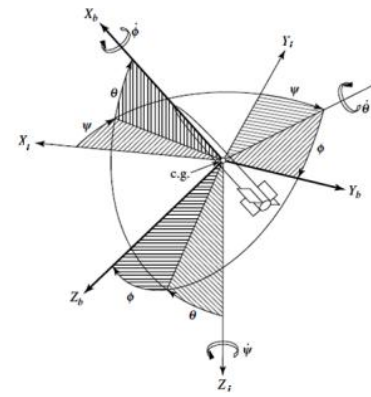

a) ICS with BCS

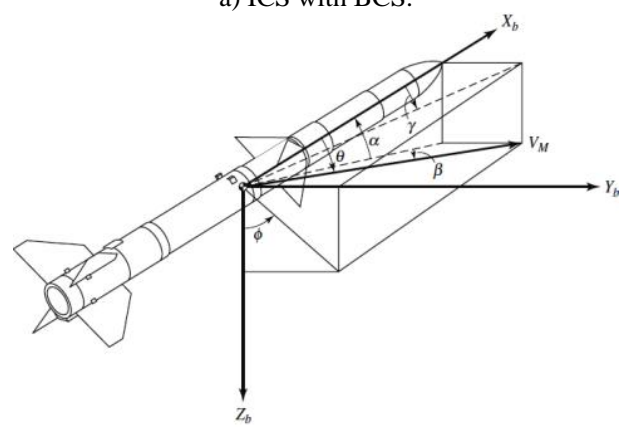

b) Velocity coordinate system with BCS.

Fig. 1. Coordinate systems orientation, and their angular relations.

$$
\begin{gathered}
\dot{u}=\frac{F_{x b}}{m(t)}+r v-q w, \quad \dot{v}=\frac{F_{y b}}{m(t)}-r u, \quad \dot{w}=\frac{F_{z b}}{m(t)}+q u \\
\dot{q}=\frac{s Q\left(h, V_{m}\right) l}{I_{y y}(t)}\left\lfloor C_{m \alpha} \alpha+C_{m \delta p} \delta_{p}+\frac{C_{m q} q l}{2 V_{m}}\right\rfloor \\
\dot{r}=\frac{s Q\left(h, V_{m}\right) l}{I_{z z}(t)}\left\lfloor C_{n \beta} \beta+C_{n \delta y} \delta_{y}+\frac{C_{n r} r l}{2 V_{m}}\right\rfloor \\
\dot{\theta}=q
\end{gathered}
$$

$$
\dot{\alpha}=q+\frac{1}{m(t) V_{m} \cos \beta}\left[F_{z b} \cos \alpha-F_{x b} \sin \alpha\right]-r \sin \alpha \tan \beta
$$$$
\dot{\beta}=-r \cos \alpha+\frac{1}{m(t) V_{m}}\left[F_{y b} \cos \beta-F_{x b} \cos \alpha \sin \beta-F_{z b} \sin \alpha \sin \beta\right]
$$

$$
\dot{h}=V_{m} \sin (\theta-\alpha)
$$

with

$$
\begin{gathered}
V_{m}=\sqrt{u^{2}+v^{2}+w^{2}}, Q\left(h, V_{m}\right)=0.5 \rho(h) V_{m}^{2} \\
F_{x b}=T_{x}(t)-s Q\left(h, V_{m}\right) C_{x}-m(t) g \sin \theta \\
F_{y b}=-s Q\left(h, V_{m}\right)\left[C_{\beta} \beta+C_{\delta y} \delta_{y}\right]
\end{gathered}
$$




$$
F_{z b}=-s Q\left(h, V_{m}\right)\left[C_{\alpha} \alpha+C_{\delta_{p}} \delta_{p}\right]+m(t) g \cos \theta
$$

where $s$ is the aerodynamic reference area in $\mathrm{m}^{2}, \rho_{0}$ is the air density at mean sea level in $\mathrm{kg} / \mathrm{m}^{3}, l$ is the missile characteristic length in $\mathrm{m}, g$ is the gravity acceleration in $\mathrm{m} / \mathrm{s}^{2}$, $T_{x}(t)$ is the function of thrust component in $X_{b}$ axis in $\mathrm{N}, m(t)$ is the function of missile mass in $\mathrm{kg}, C_{x}$ is the drag force derivative. $C_{m o}, C_{m q}, C_{m \delta p}, C_{n \beta}, C_{n r}$, and $C_{n \delta y}$, are stability derivatives of the pitching, and yawing moments, respectively. $C_{\alpha}, C_{\delta p}, C_{\beta}$, and $C_{\delta y}$, are force derivatives of the pitching, and yawing forces, respectively. $\delta_{p}$, and $\delta_{y}$ are fin angular deflections in pitch, and yaw, respectively in rad. $t$ is the flight time in sec.

Since the missile performs STT maneuver, then the missile outputs to be controlled $\left(a_{z}\right.$, and $\left.a_{y}\right)$ can be defined as follows:

$$
a_{z}=\frac{F_{z b}}{m(t)}, \quad a_{y}=\frac{F_{y b}}{m(t)}
$$

\section{B. Aerodynamic Coefficients}

Obviously, accurate estimation of the aerodynamic coefficients is the corner stone in the design of guidance and control system. Furthermore, evaluation of aerodynamic coefficients via wind tunnel tests is essential. The coefficients of aerodynamic forces, and moments obtained from aerodynamic coefficients' database based on experimental data, are functions of Mach number $M, \alpha, \beta, \delta_{p}$, and $\delta_{y}$.

\section{Environmental Conditions Dynamics Modeling}

The Lapse rate mathematical model for the troposphere has been used to represent the dynamics of air density, and the sound speed as a function of $h$ as follows:

$$
\rho(h)=\rho_{o}\left(1-\frac{L}{T_{o}} h\right)^{\frac{g}{L R}-1}, V_{s}(h)=\sqrt{\gamma R\left(T_{o}-L h\right)}, M=\frac{V_{m}}{V_{s}(h)}
$$

where $\rho(h)$ is the function of air density at altitude $h$ in $\mathrm{kg} / \mathrm{m}^{3}$, $L$ is the Lapse rate in degrees Kelvin/m, $T_{o}$ is the absolute temperature at mean sea level in degrees Kelvin, $V_{s}(h)$ is the function of speed of sound at altitude $h$ in $\mathrm{m} / \mathrm{s}, \gamma$ is the characteristic gas constant in $\mathrm{J} / \mathrm{kg}$-degrees Kelvin, and $R$ is the specific heat ratio.

\section{Autopilot Design}

In this section, the design process of pitch-yaw acceleration autopilot based on SMC, and the closed loop stability analysis are presented. The acceleration autopilot design includes outer-loop autopilot design based on SMC with acceleration dynamics modeling, inner-loop autopilot design based on SMC, and the elimination of the chattering problem.

\section{A. Pitch-Yaw Acceleration Autopilot Design}

As a result of applying the acceleration autopilot to missile model with rapid parameters' variation, a sliding mode acceleration autopilot is suggested as a robust autopilot. The scheme of the proposed acceleration autopilot based on SMC is shown in Fig. 2. The suggested acceleration autopilot consisting of a two-loop structure, controls pitch and yaw acceleration simultaneously. A novel proportional gain $K_{a c c}$ that changes according to time of the boosting phase, is added to improve autopilot tracking performance. The outer-loop of the proposed autopilot named acceleration loop generates missile pitch, and yaw angular rate commands $\left(q_{c}, r_{c}\right)$ corresponding to missile pitch, and yaw acceleration commands $\left(a_{z c}, a_{y c}\right)$. The inner-loop named rate loop generates $\left(\delta_{p}, \delta_{y}\right)$ for $\left(q_{c}, r_{c}\right)$.

\section{1) Outer-loop autopilot design based on SMC}

The derivative of the pitch acceleration can be described as:

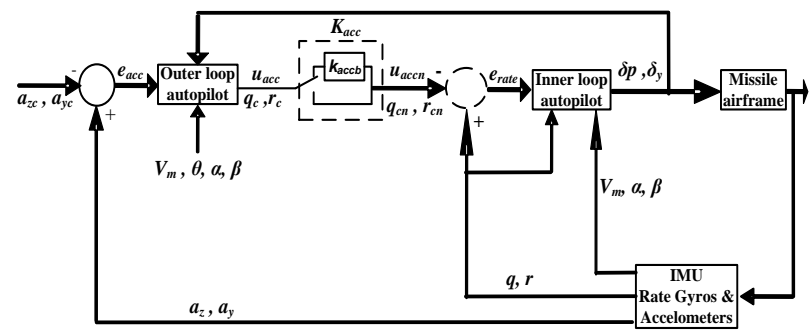

Fig. 2. Block diagram of the acceleration autopilot.

$$
\begin{aligned}
& \dot{a}_{z}=\frac{\dot{F}_{z b}}{m(t)}-\frac{\dot{m}}{m^{2}(t)} F_{z b}=-\frac{s C_{\alpha}}{m(t)} Q \dot{\alpha}-\frac{s C_{\alpha}}{m(t)} \dot{Q} \alpha-\frac{s C_{\delta p}}{m(t)} \dot{Q} \delta_{p} \\
& -\frac{s C_{\delta p}}{m(t)} Q \dot{\delta}_{p}-g \dot{\theta} \sin \theta+\frac{\dot{m}}{m(t)} g \cos \theta-\frac{\dot{m}}{m^{2}(t)} F_{z b}
\end{aligned}
$$

Substituting from (4), and (5) into (14), one gets:

$$
\begin{aligned}
& \dot{a}_{z}=-\frac{s C_{\alpha}}{m(t)} Q q-\frac{s C_{\alpha} Q}{m^{2}(t) V_{m} \cos \beta}\left[F_{z b} \cos \alpha-F_{x b} \sin \alpha\right]+\frac{s C_{\alpha}}{m(t)} Q r \sin \alpha \tan \beta \\
& -\frac{s C_{\alpha}}{m(t)} \dot{Q} \alpha-\frac{s C_{\delta_{p}}}{m(t)} \dot{Q} \delta_{p}-\frac{s C_{\delta_{p}}}{m(t)} Q \dot{\delta}_{p}-g q \sin \theta+\frac{\dot{m}}{m(t)} g \cos \theta-\frac{\dot{m}}{m^{2}(t)} F_{s b}
\end{aligned}
$$

For simplicity,

$$
\begin{aligned}
& \dot{a}_{z}=\left[N_{\alpha}-g \sin \theta\right] q+\frac{N_{\alpha}}{m(t) V_{m} \cos \beta}\left[F_{z b} \cos \alpha-F_{x b} \sin \alpha\right] \\
& -\left[N_{\alpha} \sin \alpha \tan \beta\right] r+D_{\alpha} \alpha+D_{\delta_{p}} \delta_{p}+N_{\delta_{p}} \dot{\delta}_{p}+\frac{\dot{m}}{m(t)} g \cos \theta-\frac{\dot{m}}{m^{2}(t)} F_{z b}
\end{aligned}
$$

where

$$
N_{\alpha}=-\frac{s C_{\alpha}}{m(t)} Q, \quad N_{\delta_{p}}=-\frac{s C_{\delta_{p}}}{m(t)} Q, \quad D_{\alpha}=-\frac{s C_{\alpha}}{m(t)} \dot{Q}, \quad D_{\delta_{p}}=-\frac{s C_{\delta_{p}}}{m(t)} \dot{Q}
$$

Similarly, yaw acceleration derivative is obtained as follows:

$$
\begin{aligned}
& \dot{a}_{y}=\frac{\dot{F}_{y b}}{m(t)}-\frac{\dot{m}}{m^{2}(t)} F_{y b}=-\frac{s C_{\beta}}{m(t)} Q \dot{\beta}-\frac{s C_{\beta}}{m(t)} \dot{Q} \beta \\
& -\frac{s C_{\delta y}}{m(t)} \dot{Q} \delta_{y}-\frac{s C_{\delta y}}{m(t)} Q \dot{\delta}_{y}-\frac{\dot{m}}{m^{2}(t)} F_{y b}
\end{aligned}
$$

Substituting from (6) into (17), one gets:

$$
\begin{aligned}
& \dot{a}_{y}=-\frac{s C_{\beta} Q}{m^{2}(t) V_{m}}\left[F_{y b} \cos \beta-F_{x b} \cos \alpha \sin \beta-F_{z b} \sin \alpha \sin \beta\right] \\
& +\frac{s C_{\beta} Q}{m(t)} r \cos \alpha-\frac{s C_{\beta}}{m(t)} \dot{Q} \beta-\frac{s C_{\delta y}}{m(t)} \dot{Q} \delta y-\frac{s C_{\delta y}}{m(t)} Q \dot{\delta}_{y}-\frac{\dot{m}}{m^{2}(t)} F_{y b}
\end{aligned}
$$


For simplicity,

$$
\begin{aligned}
& \dot{a}_{y}=\frac{N_{\beta}}{m(t) V_{m}}\left[F_{y b} \cos \beta-F_{x b} \cos \alpha \sin \beta-F_{z b} \sin \alpha \sin \beta\right] \\
& -N_{\beta} r \cos \alpha+D_{\beta} \beta+D_{\delta y} \delta_{y}+N_{\delta y} \dot{\delta}_{y}-\frac{\dot{m}}{m^{2}(t)} F_{y b}
\end{aligned}
$$

where

$$
N_{\beta}=-\frac{s C_{\beta}}{m(t)} Q, \quad N_{\delta y}=-\frac{s C_{\delta y}}{m(t)} Q, \quad D_{\beta}=-\frac{s C_{\beta}}{m(t)} \dot{Q}, \quad D_{\delta y}=-\frac{s C_{\delta y}}{m(t)} \dot{Q}
$$

Re-arranging the equations for the derivative of $a_{z}$, and $a_{y}$ into the matrix form, one gets:

$$
\left[\begin{array}{ll}
\dot{a}_{z} & \dot{a}_{y}
\end{array}\right]^{T}=f_{\text {acc }}\left(x, u_{\text {rate }}, t\right)+g_{a c c}(x, t) u_{a c c}
$$

where

$$
f_{\text {acc }}(\cdot) \in R^{2 \times 1}, \quad g_{a c c}(\cdot) \in R^{2 \times 2}, \quad u_{a c c} \in R^{2 \times 1}, x=\left[\begin{array}{llllll}
\alpha & \beta & q & r & V_{m} & \theta
\end{array}\right] \text { is }
$$

the state vector. The inverse of $g_{\text {acc }}(\cdot)$ always exist. $u_{\text {rate }}$ is the input vector.

$$
\begin{aligned}
& f_{a c c}(\cdot)=\left[\begin{array}{l}
\frac{N_{\alpha}}{m(t) V_{m} \cos \beta}\left[F_{z b} \cos \alpha-F_{x b} \sin \alpha\right]-N_{\alpha} r \sin \alpha \tan \beta \\
+D_{\alpha} \alpha+D_{\delta p} \delta_{p}+N_{\delta_{p}} \dot{\delta}_{p}+\frac{\dot{m}}{m(t)} g \cos \theta-\frac{\dot{m}}{m^{2}(t)} F_{z b} \\
\frac{N_{\beta}}{m(t) V_{m}}\left[F_{y b} \cos \beta-F_{x b} \cos \alpha \sin \beta-F_{z b} \sin \alpha \sin \beta\right] \\
+D_{\beta} \beta+D_{s,} \delta_{y}+N_{\delta,} \dot{\delta}_{y}-\frac{\dot{m}}{m^{2}(t)} F_{s b}
\end{array} \mid\right. \\
& g_{a c c}(\cdot)=\left\lfloor\begin{array}{cc}
N_{\alpha}-g \sin \theta & 0 \\
0 & -N_{\beta} \cos \alpha
\end{array}\right\rfloor, \quad u_{a c c}=\left[\begin{array}{ll}
q_{c} & r_{c}
\end{array}\right]^{T}
\end{aligned}
$$

The sliding surfaces $\sigma_{i}$, and the exponential reaching laws for the outer loop autopilot are defined as follows:

$$
\begin{gathered}
\sigma_{i}=e_{i}+\lambda_{i} \int_{0}^{t} e_{i} d \tau, \quad i=a z, \text { ay , } e_{a c c}=\left\lfloor\begin{array}{l}
e_{a z} \\
e_{a y}
\end{array}\right\rfloor=\left\lfloor\begin{array}{l}
a_{z}-a_{z c} \\
a_{y}-a_{y c}
\end{array}\right\rfloor \\
\dot{\sigma}_{i}=-K_{p i} \sigma_{i}-K_{s i} \operatorname{sign}\left(\sigma_{i}\right)
\end{gathered}
$$

where $\lambda_{i}, K_{p i}$, and $K_{s i}$ are positive real designed values.

Differentiating (23) with respect to time and applying the reaching law in (24), control law of the outer-loop autopilot is obtained as follows:

$$
u_{a c c}=\left[\begin{array}{ll}
q_{c} & r_{c}
\end{array}\right]^{T}=-g_{\text {acc }}(x, t)^{-1}\left[f_{\text {acc }}\left(x, \boldsymbol{u}_{\text {rate }}, t\right)+A_{\text {eacc }}+A_{\text {racc }}\right]
$$

where

$$
A_{\text {eacc }}=\left[\begin{array}{c}
\lambda_{a z} e_{a z}-\dot{a}_{z c} \\
\lambda_{a y} e_{a y}-\dot{a}_{y c}
\end{array}\right], \quad A_{r a c c}=\left[\begin{array}{cc}
K_{p a z} & \sigma_{a z}+K_{s a z} \operatorname{sign}\left(\sigma_{a z}\right) \\
K_{p a y} & \sigma_{a y}+K_{s a y} \operatorname{sign}\left(\sigma_{a y}\right)
\end{array}\right]
$$

As a result of severe missile parameters' variation during boosting phase, a new proportional gain $K_{a c c}$ is proposed and added to the outer loop scheme as shown in Fig. 2. This novel gain changes according to the boosting phase time $T_{b}$ as follows:

$$
K_{a c c}=\left\{\begin{array}{l}
k_{a c c b} \text { if } t \leq T_{b} \\
1 \text { if } t>T_{b}
\end{array}\right.
$$

where $k_{a c c b}<1$ is positive real designed number. $T_{b}$ is end time of the boosting phase.

It is obvious from (27) that, the outer-loop autopilot became having two control laws as follows:

$u_{a c c n}=\left[\begin{array}{c}q_{c u} \\ r_{c n}\end{array}\right]=\left\{\begin{array}{l}-k_{a c c b} g_{a c c}(x, t)^{-1}\left[f_{a c c}\left(x, u_{r a t e}, t\right)+A_{\text {eacc }}+A_{\text {racc }}\right] \text { if } t \leq T_{b} \\ -g_{a c c}(x, t)^{-1}\left[f_{a c c}\left(x, u_{\text {rate }}, t\right)+A_{\text {eacc }}+A_{\text {racc }}\right] \text { if } t>T_{b}\end{array}\right.$

\section{2) Inner-loop autopilot design based on SMC}

Re-arranging (2), and (3) for derivative of $q$, and $r$, respectively into the matrix form, one gets:

$$
\left[\begin{array}{ll}
\dot{q} & \dot{r}
\end{array}\right]^{T}=f_{\text {rate }}(x, t)+g_{\text {rate }}(x, t) u_{\text {rate }}
$$

where $f_{\text {rate }}(\cdot) \in R^{2 \times 1}, \quad g_{\text {rate }}(\cdot) \in R^{2 \times 2}, \quad u_{\text {rate }} \in R^{2 \times 1}$ The inverse of $g_{\text {rate }}(\cdot)$ always exist.

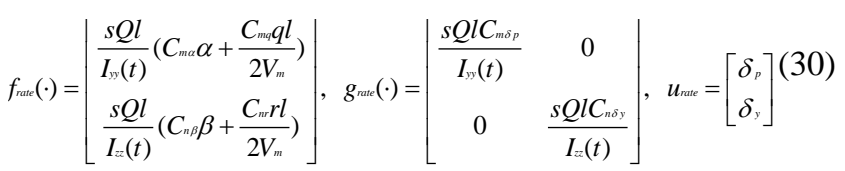

The sliding surfaces of the inner-loop autopilot are defined as:

$$
\sigma_{i}=e_{i}+\lambda_{i} \int_{0}^{t} e_{i} d \tau, \quad i=q, r \quad, \quad e_{\text {rate }}=\left\lfloor\begin{array}{l}
e_{q} \\
e_{r}
\end{array}\right\rfloor=\left\lfloor\begin{array}{c}
q-q_{c n} \\
r-r_{c n}
\end{array}\right\rfloor
$$

Differentiating Eq. (31) with respect to time and applying the reaching law in Eq. (24), control law of the inner-loop is obtained as follows:

$$
u_{\text {rate }}=\left[\begin{array}{ll}
\delta_{p} & \delta_{y}
\end{array}\right]^{T}=-g_{\text {rate }}(x, t)^{-1}\left[f_{\text {rate }}(x, t)+A_{\text {erate }}+A_{\text {rrate }}\right](32)
$$

where

$$
A_{\text {erate }}=\left[\begin{array}{c}
\lambda_{q} e_{q}-\dot{q}_{c n} \\
\lambda_{r} e_{r}-\dot{r}_{c n}
\end{array}\right], A_{\text {rrate }}=\left[\begin{array}{cc}
K_{p q} & \sigma_{q}+K_{s q} \operatorname{sign}\left(\sigma_{q}\right) \\
K_{p r} & \sigma_{r}+K_{s r} \operatorname{sign}\left(\sigma_{r}\right)
\end{array}\right]
$$

\section{3) Chattering elimination}

Unfortunately, the sign function yields to system chattering, which is considered a disadvantage for the actuator. In practice, the chattering problem can be avoided by using various methods like the boundary layer method or the continuous approximation method. In this paper, the continuous approximation method is chosen to smooth out and replace the sign function as follows [25]:

$$
\operatorname{sign}_{c a}\left(\sigma_{i}\right)=\frac{\sigma_{i}}{\left|\sigma_{i}\right|+\delta_{i}}, \quad i=a y, a z, q, r
$$

where $\delta_{i}$ is the chattering elimination parameter and it is a small positive number.

\section{B. Closed Loop Stability Study}

In order to discuss the stability of SMC based integrated pitch-yaw acceleration autopilot, The candidate Lyapunov function is defined as:

$$
V_{s}=\frac{1}{2} \sigma_{q}^{2}+\frac{1}{2} \sigma_{r}^{2}+\frac{1}{2} \sigma_{a z}^{2}+\frac{1}{2} \sigma_{a y}^{2}
$$




$$
\dot{V}_{s}=\sigma_{q} \dot{\sigma}_{q}+\sigma_{r} \dot{\sigma}_{r}+\sigma_{a z} \dot{\sigma}_{a z}+\sigma_{a y} \dot{\sigma}_{a y}
$$

Substituting (24) into (36) yields

$$
\begin{aligned}
& \dot{V}_{s}=-K_{p q} \sigma_{q}{ }^{2}-K_{s q}\left|\sigma_{q q}\right|-K_{p r} \sigma_{r}{ }^{2}-K_{s r}\left|\sigma_{r}\right| \\
& -K_{p a z} \sigma_{a z}{ }^{2}-K_{s a z}\left|\sigma_{a z}\right|-K_{p a y} \sigma_{a y}{ }^{2}-K_{s a y}\left|\sigma_{a y}\right|
\end{aligned}
$$

From Laypunov stability theorem, the appropriate choice of $K_{p q}, K_{s q}, K_{p r}, K_{s r}, K_{p a z}, K_{s a z}, K_{p a y}$, and $K_{s a y}>0$ can guarantee asymptotic stability of the sliding surfaces $\sigma_{i}, i=q, r, a z, a y$. i.e. $\dot{V}_{s}<0$. Since the only way to nullify $\sigma_{i}$ is to enforce $e_{i}=0$, tracking is carried out.

\section{NUMERICAL SIMULATIONS}

To verify performance of the proposed acceleration autopilot, numerical simulation is carried out in a MATLAB®-Simulink@ environment with step time $0.02 \mathrm{sec}$ (applicable in the real system) for the whole flight time (12 seconds) considering all missile parameters' variations in evaluating the proposed autopilot. Values of the parameters describing the airframe and the environmental conditions are listed in Table I. In particular, the missile parameters' variation will be described in details in the following section.

\section{A. Missile Dynamical Characteristics}

The underlying missile is aerodynamically controlled using canard fins. The missile contains a two stages rocket motor with boosting and sustaining phases. During boosting phase, missile acceleration is extremely high and because of that, its velocity increases from $27.4[\mathrm{~m} / \mathrm{sec}]$ up to $420[\mathrm{~m} / \mathrm{sec}]$ at 1.77 seconds as shown in Fig. 3. Obviously, excessive dynamics in missile velocity produces a great challenge to the acceleration autopilot. In the same context, dynamics of the remaining parameters describing the missile airframe are presented in details as follows [26], [27]:

\begin{tabular}{cccc}
\multicolumn{4}{c}{ TABLE I: MISSILE AND ENVIRONMENTAL CONDITIONS PARAMETERS } \\
\hline \hline Symbol & Definition & Value & Unit \\
\hline$l$ & Diameter & 0.071 & $\mathrm{~m}$ \\
$s$ & Reference area & 0.0039 & $\mathrm{~m}^{2}$ \\
$\rho_{o}$ & Air density at mean sea level & 1.2255 & $\mathrm{~kg} / \mathrm{m}^{3}$ \\
$T_{o}$ & Absolute temperature at mean sea level & 288.15 & $\mathrm{~K}$ \\
$L$ & Lapse rate & 0.0065 & $\mathrm{~K} / \mathrm{m}$ \\
$R$ & Specific heat ratio & 1.4 & - \\
$\gamma$ & Characteristic gas constant & 287.05 & $\mathrm{~J} / \mathrm{kg} / \mathrm{K}$ \\
\hline \hline
\end{tabular}

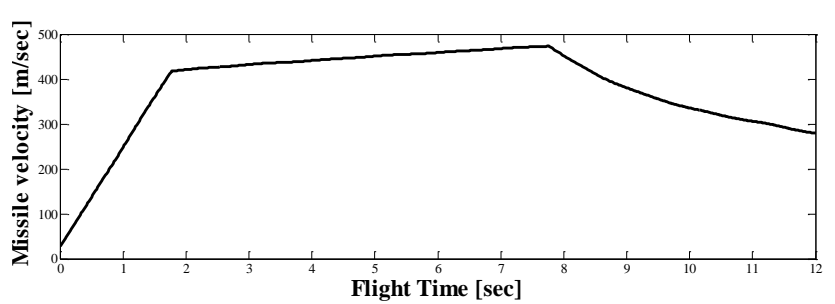

Fig. 3. Missile velocity profile.

\section{1) Aerodynamic coefficients}

Aerodynamic coefficients have in general nonlinear dependence on $M$ and on incidence angles as well. All the aerodynamic coefficients are extracted from the database at each time step.

\section{2) Missile thrust}

Thrust profile of the missile containing a two stages rocket motor with boosting and sustaining phases, is shown in Fig. 4 according to the following relation:

$$
T_{x}(t)= \begin{cases}2001.6 & \text { if } t \leq T_{b}, T_{b}=1.77 \\ 556 & \text { if } t \leq T_{s}, T_{s}=7.77 \\ 0 & \text { if } t>T_{s}\end{cases}
$$

where $T_{s}$ is end time of the sustaining phase.

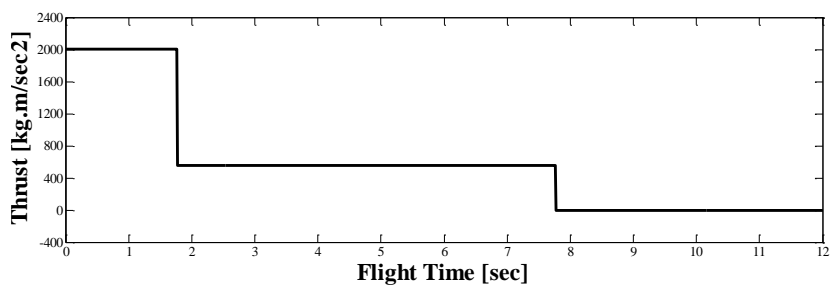

Fig. 4. Missile thrust profile.

\section{3) Missile mass}

According to missile thrust profile, mass is decreasing with time from $9.12[\mathrm{Kg}]$ to $5.25[\mathrm{Kg}]$ at the end of about 11 seconds flight. In addition, the rate of mass changes during boosting phase is faster than that during sustaining phase as shown in Fig. 5 according to the following relation:

$$
m(t)= \begin{cases}-0.98 t+9.12 & \text { if } t \leq T_{b} \\ -0.36 t+8.02 & \text { if } t \leq T_{s} \\ 5.25 & \text { if } t>T_{s}\end{cases}
$$

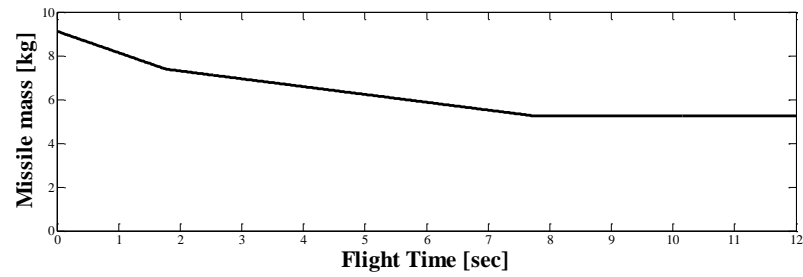

Fig. 5. Missile mass profile.

\section{4) Missile moments of inertia}

As a result of the missile mass variation with time, moments of inertia are likewise varying with time. Moreover, the missile symmetry around its longitudinal axis yields to the equivalence between $I_{y y}$ and $I_{z z}$. Typical moments of inertia for underlying missile are shown in Fig. 6; where it is approximated as a time function as long as that missile mass is always known at each instant during its flight as:

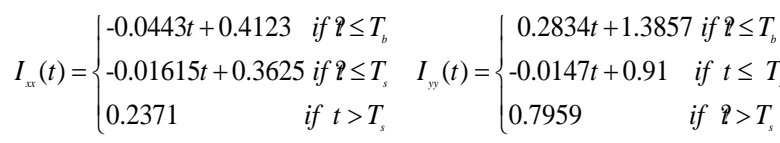

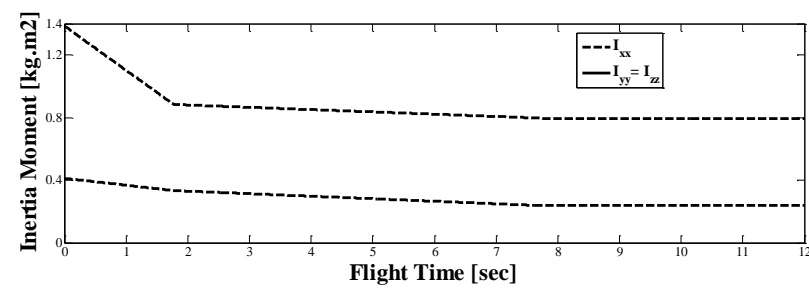

Fig. 6. Missile moments of inertia profile. 


\section{5) Hardware limitations and initial conditions}

In order to reflect physical restrictions of the mechanical system, upper and lower limits of the actuator deflection dynamics were constrained as:

$$
\max \left\{\left|\delta_{p}\right|\right\} \leq 15^{\circ}, \quad \max \left\{\left|\delta_{y}\right|\right\} \leq 15^{\circ}
$$

Initial values of the differential equations are chosen as real initial values of the underlying missile as follows:

$$
\begin{aligned}
& {\left[\begin{array}{lllllllll}
u(0) & v(0) & w(0) & q(0) & r(0) & \theta(0) & \alpha(0) & \beta(0) & h(0)
\end{array}\right]^{T}} \\
& =\left[\begin{array}{lllllllll}
27.4 & 0 & 0 & 0 & 0 & 0.5 & 0 & 0 & 2
\end{array}\right]^{T}
\end{aligned}
$$

\section{B. Acceleration Autopilot Design Parameters}

Designed parameters of the proposed pitch-yaw acceleration autopilot are chosen as follows:

$\lambda_{a z}=1000, \quad \lambda_{a y}=2000, \quad K_{p a z}=0.001, \quad K_{p a y}=0.01, \quad K_{s a z}=1$, $K_{\text {say }}=0.001, \quad \delta_{a z}=0.001, \quad \delta_{a y}=0.001, \quad k_{a c c b}=0.001, \quad \lambda_{q}=100$, $\lambda_{r}=100, K_{p q}=0.01, K_{p r}=0, K_{s q}=1, K_{s r}=1, \delta_{q}=0.01$, and $\delta_{r}=0.01$.

\section{Results}

To investigate the acceleration autopilot performance against the dynamic acceleration commands during the entire flight time, the reference pitch and yaw acceleration commands are considered as:

$$
\left[\begin{array}{ll}
a_{y c}(t) & a_{z c}(t)
\end{array}\right]^{T}=\left[\begin{array}{ll}
A_{y} \sin \omega_{y} t & A_{z} \sin \omega_{z} t
\end{array}\right]^{T}, t \geq 0
$$

where $A_{y}=5 \mathrm{~g}, A_{z}=5 \mathrm{~g}, \omega_{y}=3.14 \mathrm{rad} / \mathrm{sec}, \omega_{z}=3.14 \mathrm{rad} / \mathrm{sec}$.

The effect of adding the novel gain $K_{a c c}$ to the autopilot on the acceleration tracking performance and the tracking error in yaw, and pitch planes are depicted in Fig. 7-10, respectively. Fig. 7-10 show that the gain $K_{a c c}$ enhances tracking performance, minimizes tracking error, and reduces overshoots in both yaw, and pitch channels, especially in the boosting phase. The gain $K_{a c c}$ minimizes both angles $\alpha$, and $\beta$ and their variations as shown in Fig. 11, 12, respectively. The smallness of $\alpha$, and $\beta$ minimizes aerodynamic non-linearity and relaxes the coupling between planes of symmetry. On the other hand, the gain $K_{a c c}$ produces a great smoothing in both yaw, and pitch fin deflections as shown in Fig. 13-14, respectively, which relaxes the actuator and minimizes the control efforts. The fin deflections increase during gliding phase to overcome the decrement of aerodynamic forces and moments due to missile velocity reduction. It is evident that, overall simulation results of the proposed autopilot have shown a good tracking performance in presence of all the missile parameters' variation, the environmental conditions' dynamics, the dynamic acceleration commands in boosting phase, and the rapid velocity variation.

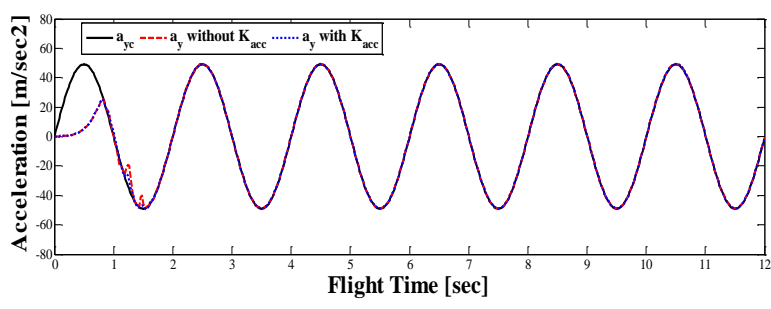

Fig. 7. Yaw acceleration profile without/with $K_{a c c}$ gain.

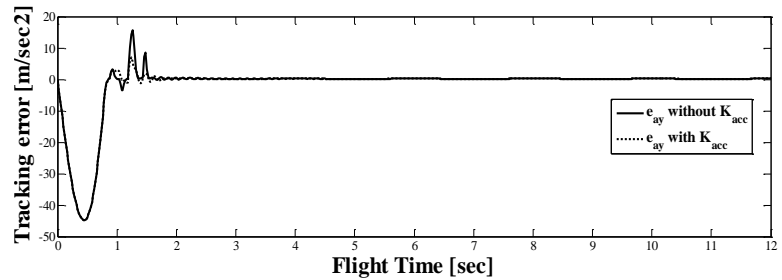

Fig. 8. Yaw acceleration tracking error profile without/with $K_{a c c}$ gain.

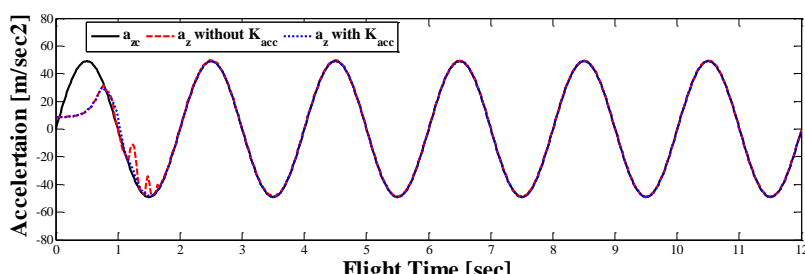

Fig. 9. Pitch acceleration profile without/with $K_{a c c}$ gain.

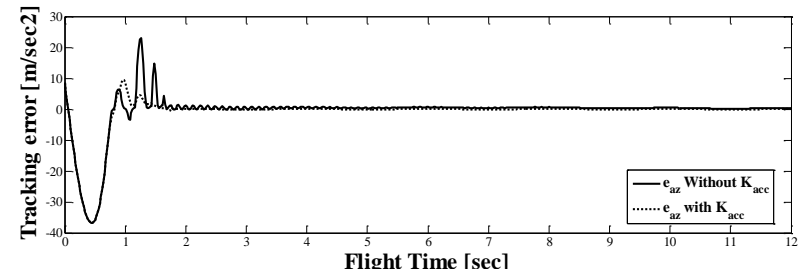

Fig. 10. Pitch acceleration tracking error profile without/with $K_{a c c}$ gain

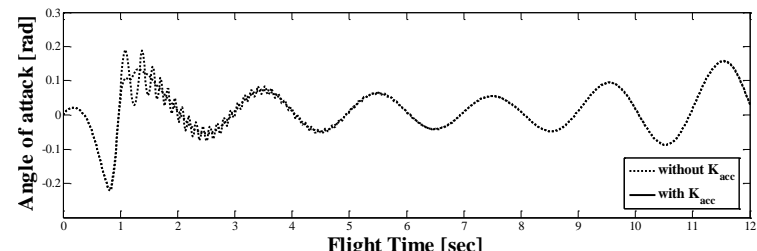

Fig. 11. Angle of attack profile without/with $K_{a c c}$ gain.

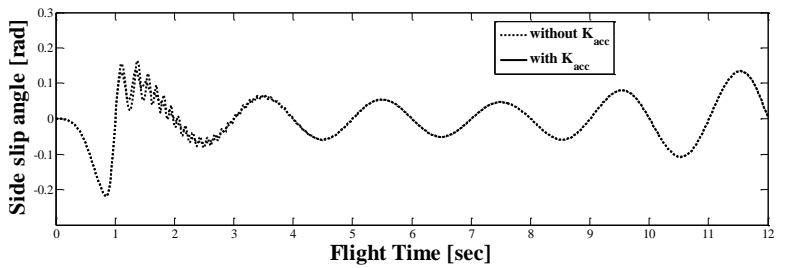

Fig. 12. Side slip angle profile without/with $K_{a c c}$ gain.

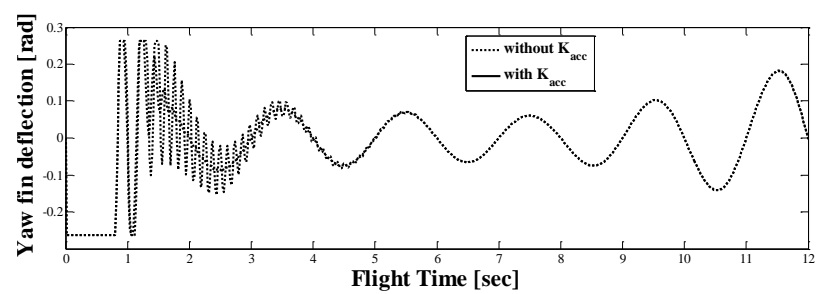

Fig. 13. Yaw fin deflection profile without/with $K_{a c c}$ gain.

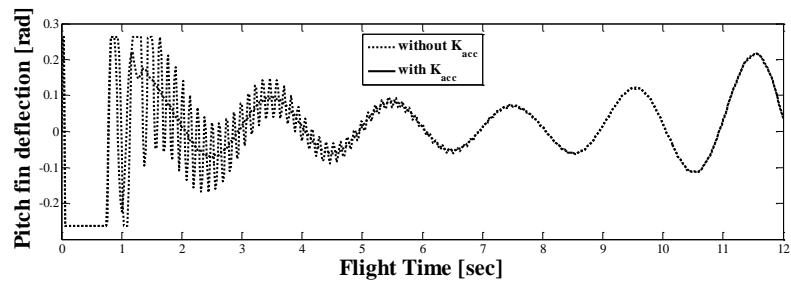

Fig. 14. Pitch fin deflection profile without/with $K_{a c c}$ gain.

\section{CONCLUSION}

In this paper, sliding mode control based integrated 
pitch-yaw acceleration autopilot design has been presented for roll-stabilized skid-to-turn varying-velocity man-portable missile by adding a switched gain to the autopilot outer loop to improve the tracking performance and to overcome the undesirable effects occurring due to the considerations of the common disregarded factors represented in the gravity effect, dynamic acceleration commands in boosting phase, and more missile model dynamics during the entire flight time. The closed loop stability analysis is performed. Numerical simulation is conducted to verify the proposed autopilot performance, and its results show robustness and good performance in the presence of the aforesaid considerations. The suggested future work is considering the coupling effect between missile channels due to the rolling motion.

\section{REFERENCES}

[1] G. Mattei and S. Monaco, "Nonlinear autopilot design for an asymmetric missile using robust back stepping control," Journal of Guidance, Control, and Dynamics, vol. 35, no. 5, 2014, pp. 1462-1476.

[2] Y. Lee, Y. Kim, G. Moon, and B. Jun, "Sliding mode based attitude and acceleration controller for a velocity-varying skid-to-turn missile," European Control Conference, 2014, pp. 2951-2956.

[3] X. Chen, J. Song, and G. Cai, "Robust gain-scheduled autopilot design with LPV reference model for portable missile," in Proc. 2012 IEEE Aerospace Conference, 2012, pp. 1-10.

[4] P. Zarchan, Tactical and Strategic Missile Guidance, AIAA, Reston, VA, 2007, pp. 507-527.

[5] F. W. Nesline, B. H. Wells, and P. Zarchan, "Combined optimal/ classical approach to robust missile autopilot design," Journal of Guidance, Control, and Dynamics, vol. 4, no. 3, 1981, pp. 316-322.

[6] J. S. Shamma and J. R. Cloutier, "Gain-scheduled missile autopilot design using linear parameter varying transformations," Journal of Guidance, Control, and Dynamics, vol. 16, no. 2, 1993, pp. 256-263.

[7] R. A. Nichols, R. T. Reichert, and W. J. Rugh, "Gain scheduling for $H \infty$ controllers: A flight control example," IEEE Transactions on Control Systems Technology, vol. 1, no. 2, 1993, pp. 69-79.

[8] V. Fromion, S. Monaco, and D. Normand-Cyrot, "The weighted incremental norm approach: From linear to nonlinear $H_{\infty}$ control," Automatica, vol. 37, no. 10, 2001, pp. 1585-1592.

[9] A. Mahmood, Y. Kim, and J. Park, "Robust $H \infty$ autopilot design for agile missile with time-varying parameters," IEEE Transactions on Aerospace and Electronic Systems, vol. 50, no. 4, 2014, pp. 3082-3089.

[10] W. Tan, A. K. Packard, and G. J. Balas, "Quasi-LPV modeling and LPV control of a generic missile," in Proc. the 2000 American Control Conference, 2000, pp. 3692-3696.

[11] M. Sato, "Filter design for LPV systems using quadratically parameter-dependent lyapunov functions," Automatica, vol. 42, no. 11, 2006, pp. 2017-2023

[12] C. Schumacher and P. P. Khargonekar, "Stability analysis of a missile control system with a dynamic inversion controller," Journal of Guidance, Control, and Dynamics, vol. 21, no. 3, 1998, pp. 508-515.

[13] E. Devaud, H. Siguerdidjane, and S. Font, "Some control strategies for a high-angle-of-attack missile autopilot," Control Engineering Practice, vol. 8, no. 8, 2000, pp. 885-892.

[14] E. V. Oort, L. Sonneveldt, Q. Chu, and J. Mulder, "Modular adaptive input-to-state stable backstepping of a nonlinear missile model," in Proc. AIAA Guidance, Navigation and Control Conference and Exhibit, 2007.

[15] C. Cao and N. Hovakimyan, " $l_{l}$ adaptive output-feedback controller for non-strictly-positive-real reference systems: Missile longitudinal autopilot design," Journal of Guidance, Control, and Dynamics, vol. 32, no. 3, 2009, pp. 717-726.

[16] I. Rusnak, H. Weiss, and I. Barkana, "Improving the performance of existing missile autopilot using simple adaptive control," Int. J. Adapt. Control Signal Process, vol. 28, 2014, pp. 732-749.

[17] K. W. Lee and S. N. Singh, "Noncertainty-equivalent adaptive missile control via immersion and invariance," Journal of Guidance, Control, and Dynamics, vol. 33, no. 3, 2010, pp. 655-665.

[18] N. Pal, R. Kumar, and M. Kumar, "Design of missile autopilot using backstepping controller," in Proc. International Conference on Electronics, Communication and Instrumentation (ICECI), 2014, pp. $1-4$.

[19] T. Cimen, "A generic approach to missile autopilot design using state-dependent nonlinear control," in Proc. 18th IFAC World Congress, vol. 18, no. 1, 2011, pp. 9587-9600.

[20] A. Thukral and M. Innocenti, "A sliding mode missile pitch autopilot synthesis for high angle of attack maneuvering," IEEE Transactions on Control Systems Technology, vol. 6, no. 3, 1998, pp. 359-371.

[21] S. Kang, H. J. Kim, J. I. Lee, B. E. Jun, and M. J. Tahk, "Roll-pitch-yaw integrated robust autopilot design for a high angle-of-attack missile," Journal of Guidance, Control, and Dynamics, vol. 32, no. 5, 2009, pp. 1622-1628.

[22] E. Devaud, H. Siguerdidjane, and S. Font, "Three-axes missile autopilot design: From linear to nonlinear control strategies," Journal of Guidance, Control, and Dynamics, vol. 24, no. 1, 2001, pp. 64-71.

[23] G. M. Siouris, Missile Guidance and Control Systems, Springer-Verlag, New York, Inc., 2004.

[24] P. Garnell and D. J. East, Guided Weapon Control Systems, 2nd ed., New York, 1980, Chaps. 3, 4.

[25] X. Yu and O. Kaynak, "Sliding-mode control with soft computing: A survey," IEEE Transactions on Industrial Electronics, vol. 56, no. 9, 2009, pp. 3275-3285.

[26] M. Medjaoui, "Performance evaluation of short range IR homing heads in the presence of countermeasures," M. Sc. Thesis, Department of guidance, M.T.C., Cairo, 1992.

[27] A. Awad and H. Wang, "Evaluation and enhancing missile performance via real time flight simulation model," Chinese Control Conference, 2014, pp. 6324-6329.

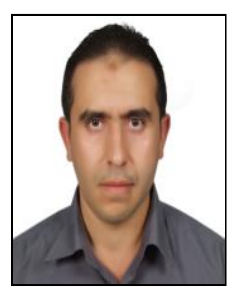

A. Awad received his B.Sc. in electric engineering, with grade excellent with honor, Cairo, Egypt, in 2002; technical support engineer until 2004, senior researcher until 2006. In 2010, he has received the M.Sc. degree in electric engineering, in missile infrared seeker and flight simulation, Military Technical College, Cairo, Egypt. He is interested in embedded systems and interfaces, real time systems, hardware-in-loop simulation, visual tracking, and mostly concerned in missile guidance and control. Currently, he is a Ph.D. candidate in Nanjing University of Science and Technology (NUST) at School of Automation, Nanjing, Jiangsu, China.

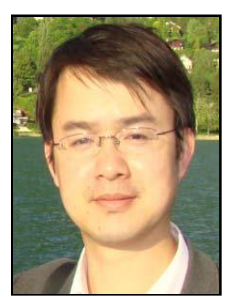

H. Wang received his Ph.D. degree in automatic control from Lille University of Science and Technology (LUST), France, in 2008. He is currently professor at Automation School, Nanjing University of Science and Technology, China. He was research fellows at Modeling Information and System Laboratory of Picardie University and at Automatics, Computer Engineering and Signal Processing Laboratory of LUST, France. His research interests include the theory and applications of hybrid systems, visual servo control, friction modeling and compensation, modeling and control of diesel engines, biotechnological processes and wind turbine systems. 